\section{Extreme Rainfall Multiplier An Intuitive Metric for Tropical Cyclone Hazards}

Adapted from "An Intuitive Metric to Quantify and Communicate Tropical Cylcone Rainfall Hazard," by C. D. Bosma (University of Wisconsin-Madison), D. B. Wright, P. Nguyen, J. P. Kossin, D. C. Herndon, and J. M. Shepherd. Published Online in BAMS, February 2020. For the full citable article, see DOI:10.1175/BAMS-D-19-0075.1.

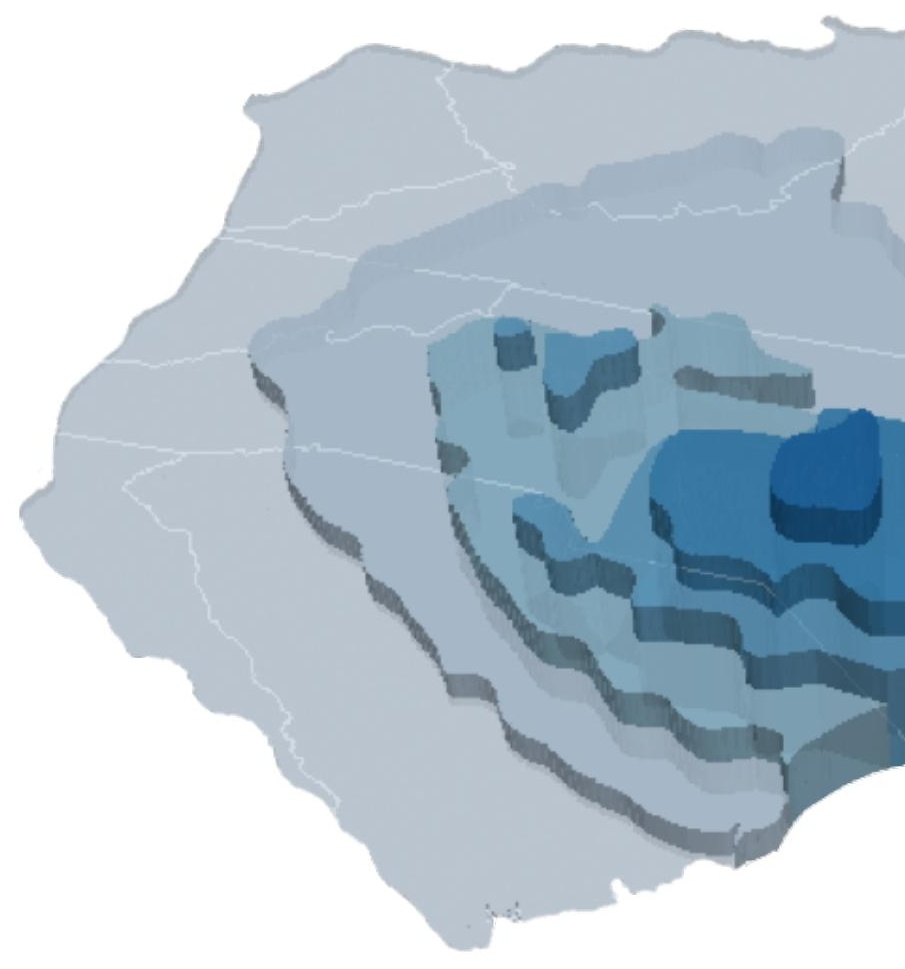

c

limate models have indicated that tropical cyclone (TC) rainfall rates in the North Atlantic will increase. Already, the mean translational speed of North Atlantic TCs over land has decreased by $20 \%$ since the mid-twentieth century. Together these trends mean that more intense, slower-moving storms are more likely to linger and to generate extreme rainfall, which already (between 1963 and 2012) is the deadliest TC hazard in the United States.

The widely used Saffir-Simpson scale, however, assigns TCs a hazard category from 1 to 5 based on maximum sustained wind speed. This is a not a reliable indicator of extreme rainfall hazard. While a TC's wind speed over land is typically much weaker than over the ocean, the rainfall threat may still be high post-landfall. Additionally, the area most impacted by extreme rainfall during a particular TC may be both spatially and temporally distinct from the region with the highest winds.

Hurricane Harvey (2017) and Hurricane Florence (2018) highlighted the hazard posed by TC rainfall and the challenge of effectively communicating about it to the public, especially in terms of Saffir-Simpson categories and recurrence intervals. Hurricane Florence, for example, 
approached the East Coast of the United States as a category 4 hurricane before weakening and making landfall as a category 1 storm. The downgrade in Florence's category as it approached the coast may have been perceived as a reduced hazard, resulting in some residents choosing not to evacuate. The storm produced extreme rain-more than $900 \mathrm{~mm}$ in Elizabethtown, North Carolina-and severe flooding throughout the Carolinas. In another case the previous year, Hurricane Harvey stalled over southeastern Texas for several days, making landfall as a category 4 hurricane and weakening to a tropical storm shortly thereafter. More than 1,500 mm of rain fell in Nederland, Texas-the highest TC-related rainfall total ever recorded in the United States.

The magnitude of Hurricane Harvey's rain was communicated by the media as a "500-year" or "1,000-year" event, but much of the public is confused by these recurrence intervals. These metrics are probabilities, not literal facts. A rainfall or flood event with a 500-yr recurrence interval has a 1 in 500 (0.2\%) probability of being exceeded in any year (i.e., annual exceedance probability); such an event will occur, on average, once every 500 years in a stationary (i.e., unchanging) climate. Though highly unlikely, two 500-yr events could impact a region within a calendar year. While the likelihood of an extreme event like Hurricane Harvey occurring at any specific locationsuch as the Houston metropolitan area-is very low, the probability of an event of this magnitude occurring somewhere within the eastern United States may be considerably higher. This distinction is probably lost on many people and may contribute to further confusion when recurrence intervals are mentioned in the popular media.

Also, the understanding of probabilistic metrics is highly individual: the same metric can have different meanings for different people based on their own perception of risk. A growing number of studies highlight the importance of "experiential processing" in everyday decision-making: people often relate current situations to events they can recall from prior experience or recent media reports and images. An individual may struggle to understand the magnitude associated with a rare event (such as a 500-year rainfall), particularly if such an event has not occurred recently in their location. A survey in a floodprone community in Texas, for example, revealed residents were more concerned about a potential flooding hazard when provided with concrete information about the nature of flooding, as opposed to abstract probabilities. Preparedness ahead of high-impact floods in 2015 and 2016 in the United Kingdom may have been reduced because residents had trouble adequately conceptualizing the magnitude of the flooding, which exceeded any in recent memory.

\section{The extreme rainfall multiplier}

Given these shortcomings with existing methods of communicating about extreme rainfall, we propose a scale to

1. accurately characterize the TC rainfall hazard;

2. identify "locally extreme" events because local impacts increase with positive deviations from the local rainfall climatology;

3. succinctly describe TC rainfall hazards at a range of time scales up to the lifetime of the storm system;

4. be easy to understand and rooted in experiential processing to effectively communicate the hazard to the public.
Our metric, the extreme rainfall multiplier (ERM), is the ratio of the rainfall depth for a given storm (over a specific location and duration) to the rainfall depth (same location, duration) at a given recurrence interval.

For the recurrence interval in calculating ERM for this study, we use the 2-yr rainfall event, representing a baseline, relatively frequent "heavy" rainstorm. The 2-yr rainfall represents the median annual maximum rainfall (the median value in a time series of the largest rain events per year, based on recent climatology), which statistically has a 50\% chance of being exceeded in any given year. A "rule of thumb" is that floods or rainstorms below the 2-yr recurrence interval (e.g., ERM < 1.0) generally will not produce negative impacts, while those above the 2-yr recurrence interval (e.g., ERM > 1.0) may.

Expressing the magnitude of rare events as multiples of the 2-yr rainfall (derived from local climatology) can help the public utilize their own experiences to conceptualize the magnitude of extreme events. Such (likely recent) experience can serve as an "anchor," which, in cognitive psychology, is information upon which subsequent judgements are based.

\section{Analysis of historical tropical cyclone ERMs}

We analyzed the 385 North Atlantic TCs that made landfall or passed within $500 \mathrm{~km}$ of the eastern United States between 1948 and 2017. Precipitation was calculated using NOAA's gridded, gauge-based Climate Prediction Center (CPC)-Unified dataset, which was also used for estimating the 2-yr rainfall for each

For storm $s$ and location $x$, duration $t$, and rainfall depth $R_{s, x, t}$

$$
\mathrm{ERM}_{s, x, t}=\frac{R_{s, x, t}}{R_{x, t}^{T-\mathrm{yr}}} .
$$

The denominator $R_{x, t}^{T-y r}$ is the rainfall depth for the $T$-yr average recurrence interval for the same location and duration. In estimating the denominator, the uncertainty decreases as the period of record increases. However, rainfall observations from earlier in the CPC-Unified record may be less representative of present-day heavy rainfall. To solve this issue, we propose calculating the median (i.e., 2-yr) annual maximum rainfall for each grid cell for the 30-year period of 1981 to 2010. This median, rather than the mean, also minimizes the impact of outlier events in determining the 2-yr climate normal. 
grid cell. Using these values, we converted daily and multiday rainfall totals, up to the lifetime of each storm, into ERMs.

While the mean single-cell storm maximum ERM across these 385 storms is approximately 2.0, 19 TCs (4.8\%) have ERMs greater than 4.0, including Hurricane Harvey, which has the highest ERM (6.4) in the database.

A large majority (74\%) of storm maximum ERMs are associated with rainfall durations of 1 day, highlighting how TCs are only occasionally long-lived rainfall events. However, high ERMs occur at both daily and multiday time scales, as Hurricanes Harvey, Georges, and Floyd demonstrate. The correspondence between high ERM and high storm impacts for these three TCs, despite relatively low SaffirSimpson categories at the time of rainfall impact, lends support to the suitability of ERM as a metric for characterizing TC rainfall hazard.

\section{Regional distribution of tropical cyclone ERMs}

ERMs generally decrease with distance from the coast. However, there is a wide geographic distribution of high ERMs throughout three regions: the Texas-Louisiana area of the Gulf Coast, the rest of the Gulf Coast and the inland southeast (including Florida and Georgia), and the remaining states along the Atlantic Coast. Despite differences in the types and paths of landfalling TCs, the distributions of single-cell storm-maximum ERMs in these regions are remarkably similar. These results suggest that the distribution of ERM maxima is generally not affected by geographic location and help place hazard communication within the context of the local rainfall hydroclimate. Further, this regional invariance of ERM maxima may also enable development of models to estimate the recurrence interval of high ERMs with extensive geographic footprints. For example, the large spatial extent of Hurricane Harvey's extreme rainfall was a major contributor to its catastrophic impacts. With this information, recurrence interval estimates of ERM extent could be derived, which may have utility in forecasting or risk assessment.

\section{Using the ERM to communicate a TC rainfall forecast}

To demonstrate how ERM could be used in a forecast setting, we calculated values for Hurricane Florence from 5-day quantitative

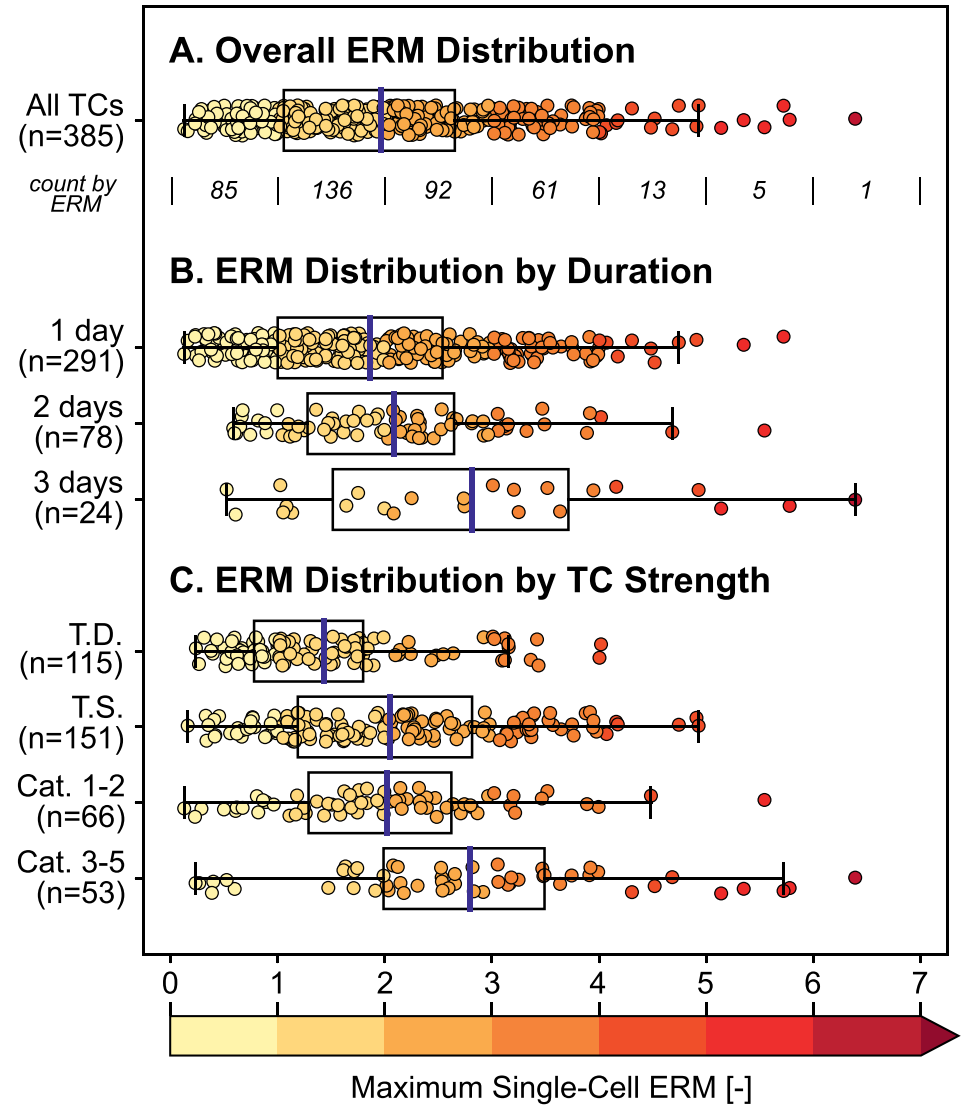

$\triangle$ Three different ways of looking at the distribution of single-cell maximum ERM (including peak TC strength within $500 \mathrm{~km}$ of initial TC landfall) for landfalls in the contiguous United States. Blue lines indicate the mean for each distribution. precipitation forecasts (QPFs) issued by NOAA's National Weather Service Weather Prediction Center (WPC). Florence weakened more rapidly than expected as it approached North Carolina, ultimately making landfall as a category 1 system. The resulting wind-related impacts, while severe, were less than originally anticipated.

In contrast to the wind hazard, forecast ERM for Hurricane Florence indicates that the expected rainfall hazard was very high, and increasing, as the storm neared the United States. Estimates of the peak, single-cell, 5-day ERM for Hurricane Florence increased from 4.2 on 12 September to 5.0 on 14 September. The estimated 3-day ERM on the day of Florence's landfall was even higher at 5.7. These extremely high forecast ERM values remained elevated amid Florence's weakening winds. A 5.7 would equal Hurricane Floyd (1999) as the highest ERM ever seen on the Atlantic coast. Additionally, though we focus here on the single-cell storm-maximum ERM, the spatial extent of rainfall hazard could be communicated to the public using forecast maps of the ERM. 

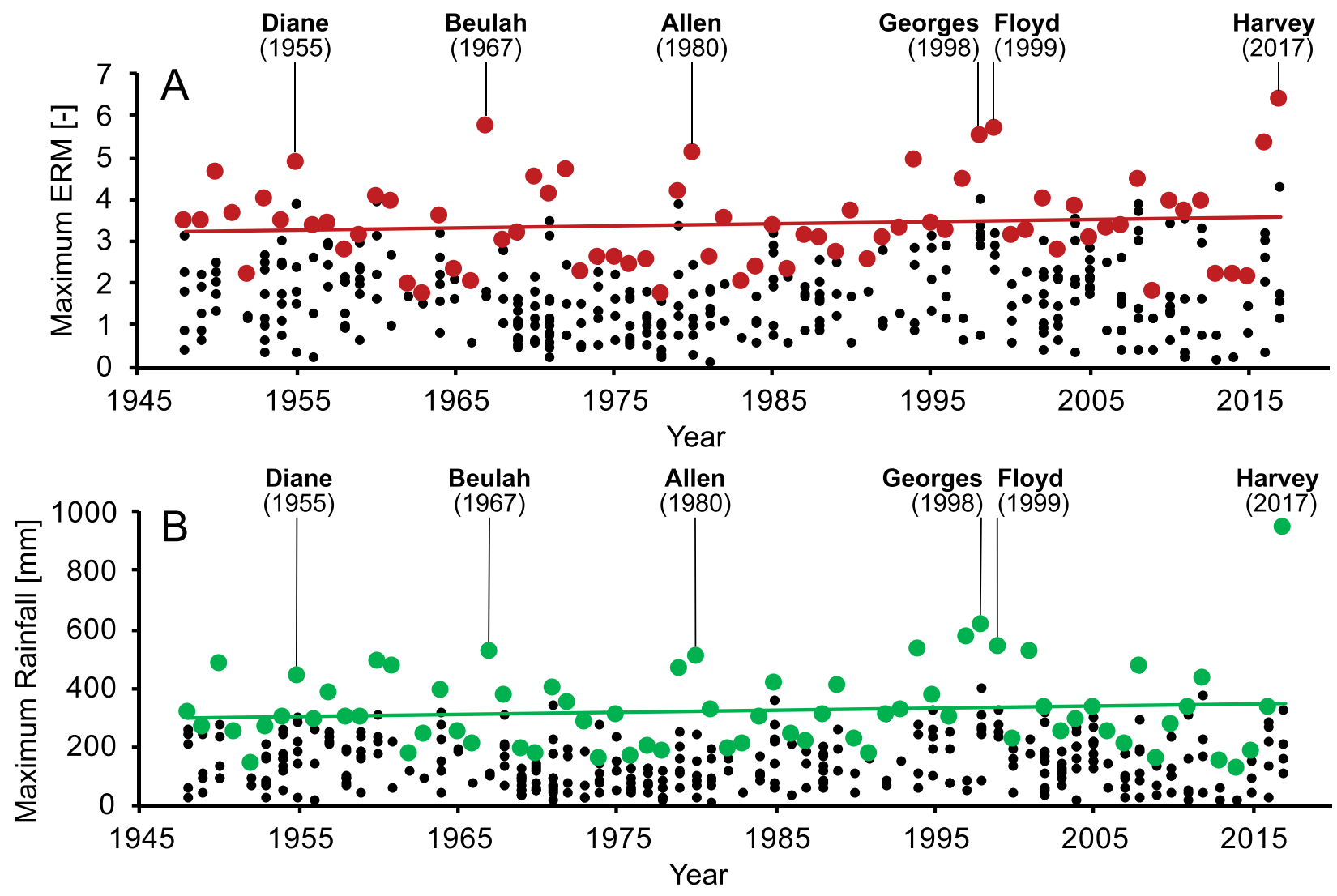

When compared to current forecast products and to recurrence interval estimates, ERM forecast values can contextualize extreme QPF values and potentially provide a more tangible meaning to the public. Comparing forecasts to similarly extreme historical ERMs might help forecasters to depict possible damages. This type of imagery has been shown to increase risk perception and highlights another way ERM could be used to identify and communicate potential rainfall hazard in the days before landfall. At least one rainfall hazard product-the Excessive Rainfall Outlook (ERO) issued by the WPC-focuses on the probability of rainfall exceeding flash flood thresholds, emphasizing the likelihood rather than the magnitude of the rainfall threat. ERM could be a complement or alternative to these outlooks.

\section{Summary and conclusions}

Despite observational evidence for increasing TC intensity and decreases in translational speed, relatively less work has been done to assess changes in TC rainfall hazards. Challenges facing TC rainfall trend studies include the limited observations available at any particular location as well as the influences of $\triangle$ Highest annual maximum ERMs (1948-2017) are indicated with colored markers, and colored lines represent linear regression fit. A Mann-Kendall test for monotonic trends in annual maxima values did not reveal significant changes over time for either ERM or rainfall. geographically varying rainfall hydroclimate and storm lifetime. The ERM framework to quantify TC rainfall offers several useful properties. The metric normalizes extreme rainfall in the context of climatology at particular locations. ERM also allows for detailed comparison of the relative severity of rainfall between locations, focusing not on the sheer magnitude of the rainfall but the deviation from more typical events. Unlike metrics such as daily amounts or deviation from normal, ERM is independent of date, allowing comparison across different times of the year. The distribution of ERM maxima is similar throughout regions of the eastern United States, allowing the development of regional-scale (rather than local-scale) recurrence interval estimates for extreme TC rainfall. With the regional invariance of ERM, one could consider all ERM events as belonging to a single population, thus increasing sample size and permitting robust regional recurrence estimation based on large geographical footprints.

When applied retrospectively, ERMs correspond with observed TC rainfall impacts for several high-impact events, confirming that it can depict hazards. ERMs reflect geographic 

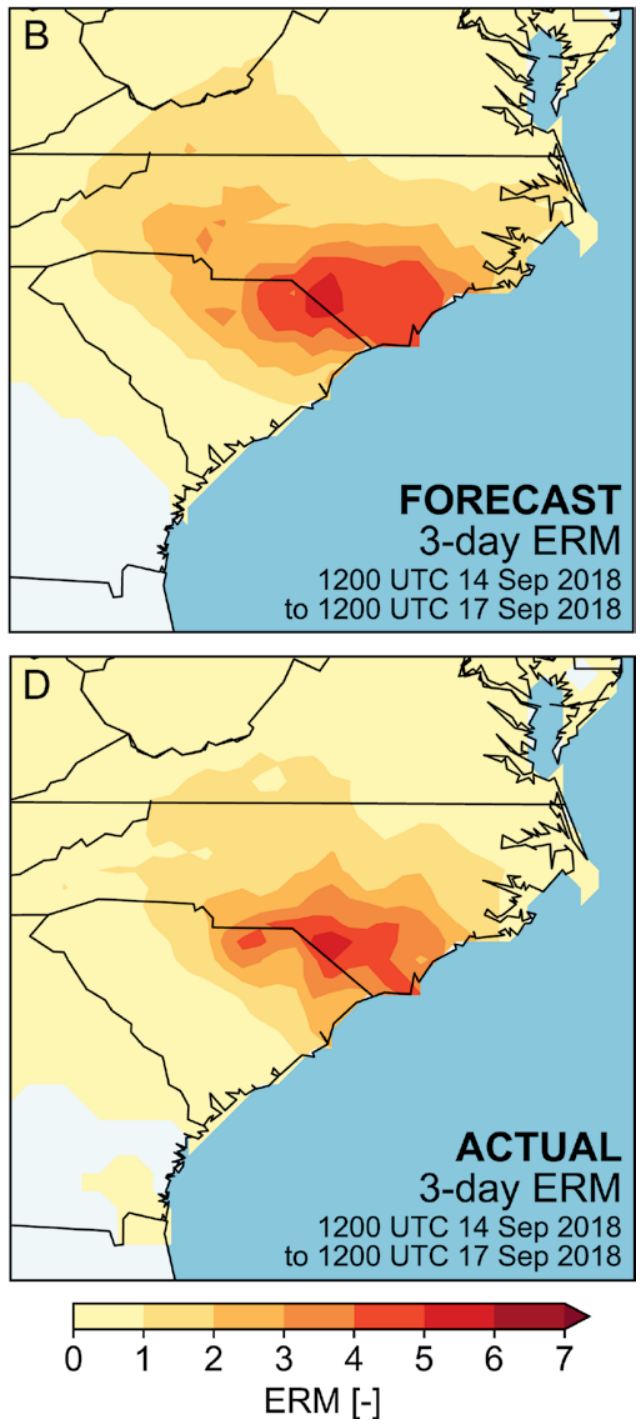

differences in the climatology of rainfall extremes and can succinctly describe TC rainfall hazards of varying durations using a single scale. Theoretically, the ERM framework could be used for other natural hazards, not just TCs.

A number of issues must be resolved to use ERM in operational forecast communications. These include the differences in the resolution and coverage of gridded rainfall products and precipitation forecasts, how and whether to communicate a range of forecast lead times or durations, and what graphical and verbal techniques communicate the information most effectively. Nonetheless, our Hurricane Florence ERM hindcast shows that the method can accurately characterize the rainfall hazard of a significant TC several days ahead in a way that can be readily communicated to, and interpreted by, the public. $\measuredangle *$ (Top) ERM forecast for Hurricane Florence post-landfall derived from NWS QPF estimates for 3-day rainfall issued at 1200 UTC 14 Sep 2018. (Bottom) 3-day ERM based on poststorm CPC-Unified data. ERM was above $\mathbf{3 . 0}$ for $36,900 \mathrm{~km}^{2}$ across the Carolinas. The utility of any ERM forecast in this context is dependent on the accuracy of the rainfall forecast used to generate ERM values.

BAMS: What would you like readers to learn from this article?

\section{J. Marshall Shepherd (University of Georgia): Messaging the deadly} water-related threat in hurricanes was a problem brought to light with Hurricanes Harvey and Florence. Our paper is offering a new approach to this critical topic using sound science methods.

Christopher Bosma: One goal of this paper is to give various stakeholders - from meteorologists to emergency planners to the media-an easy-to-understand, but statistically meaningful, way of talking about the frequency and magnitude of extreme rainfall events, particularly those caused by tropical cyclones.

BAMS: How does the problem manifest itself in warnings?

James Kossin (NOAA/NCEI): Coastal residents are warned about these hazards. Freshwater flooding from extreme hurricane rainfall, however, can happen inland, away from evacuation zones, and pose the greatest threat where people tend to shelter in place. Compound hazards such as dam failures and landslides pose additional significant threats. Effective warnings and communication of inland threats are paramount to reduce mortality.

\section{Daniel Wright (University of} Wisconsin-Madison): The usual ways that we communicate the hazards posed by hurricanes and tropical storms neglects rainfall and the flooding it can cause.

The conventional language-for example, "category 5 hurricane"correlates poorly with the risks of rainfall and freshwater flooding.

\section{Phu Nguyen (University of} California-Irvine): The SaffirSimpson hurricane wind scale 
is classified based on wind

speed, disregarding rainfall depth. Our new metric identifies and characterizes rainfall depth.

BAMS: Classifications don't always "characterize," though-how does ERM do this?

JK: The tool is for providing warnings based on people's past experience, which gives them a familiar reference point from which to assess their risk and make informed decisions.

BAMS: How does framing extreme rainfall and floods in terms of return periods contribute to the communications problem?

DW: I am trained as a civil engineer, and we worry a lot in engineering about ideas such as the "100-year flood" because it forms the basis of lots of infrastructure planning and flood risk management. However, I get nervous when I see the media, and even the president, talking about 500-year storms or floods. There are some pretty specific definitions in that terminology that the public isn't aware of. It is unfortunately too easy to give people the wrong idea about how rare and severe these events can be. So the goal was to find a better way to talk about these ideas and risks with nonexperts.

BAMS: What insights did you get from reinterpreting past storms in a new way?

CB: It was eye-opening to realize that extreme rainfall events similar to Hurricane Harvey, such as Hurricane Diane, have happened in other parts of the country.

DW: Hurricane Diane in 1955 was absolutely devastating, and ushered in a building boom of flood control dams throughout New England.

I've always been an amateur historian, and so I love to learn about the context of these earlier storms-and to see how they can help us learn about the present and future.

BAMS: What was the biggest challenge in this work?

CB: We started out focused on analyzing the catastrophic and record-breaking rainfall associated with Hurricane Harvey. But, then Hurricane Florence brought devastating and torrential rainfall to the Carolinas, which forced us to go back and revisit some of our initial analysis. The fact that multiple major storms happened in quick succession provided a chance to research the messaging used to describe these systems and think of other ways to use the metric we had developed-such as the ERM "forecast."

DW: For more than 10 years I've been reading and writing research papers for science and engineering audiences. This work was a little different because, while we wanted the science to be airtight, we needed to aim for a broader audience and needed to "keep it simple."

BAMS: What is the key to making that leap-from a metric useful in research to a way to warn the public?

JK: Definitely the greatest challenge is determining how to present the general populace with warnings that resonate with individuals and allow a sense of familiarity of the situation and threat. We had to wear two hats: one as physical scientists and another as psychologists. Most of us have much more formal training in the former than in the latter.

BAMS: Do you feel ERM meets that challenge?

DW: I'm proud of how Chris, a Ph.D. student in my group, was able to soak up ideas from different places and form it into a really solid idea that, I hope, can make a difference in how we talk about the risks of hurricane and tropical storm rainfall.

BAMS: What surprised you the most about this work?

JMS: That we produced a credible methodology for a difficult but timely challenge.

JK: I suppose the thing that surprises me most is how difficult it is to effectively communicate hazard and risk to people, and to galvanize potentially life-saving action. There's a lot of human psychology at play, and humans are an odd lot when it comes to understanding and responding to risk.

BAMS: How will you follow up?

DH: Almost certainly any follow-up work will be driven by feedback from the community. One question is: how can this approach be applied to other parts of the world where extreme rainfall from TCs poses perhaps an even greater risk? 